\title{
Practice and Attitudes of Physicians Regarding Disclosure of Information to Patients With Serious Illness
}

\author{
Ghufran Jassim ${ }^{1}$, Alaa Alakri ${ }^{2}$, Rawaa Alsayegh ${ }^{2} \&$ David Misselbrook $^{1}$ \\ ${ }^{1}$ Family Medicine Department, School of Medicine, Royal College of Surgeons in Ireland-Medical Universitry \\ of Bahrain, Busaiteen, Bahrain \\ ${ }^{2}$ School of Medicine, Royal College of Surgeons in Ireland-Medical Universitry of Bahrain, Busaiteen, Bahrain \\ Correspondence: Ghufran Jassim, School of Medicine, Royal College of Surgeons in Ireland-Medical \\ Universitry of Bahrain, Busaiteen, Bahrain. Tel: 97-33-968-0009. E-mail: gjassim@rcsi-mub.com
}

Received: February 4, 2019 Accepted: March 13, 2019 Online Published: April 3, 2019

doi:10.5539/gjhs.v11n5p33 URL: https://doi.org/10.5539/gjhs.v11n5p33

\begin{abstract}
Background: Health Information disclosure is the cornerstone in respecting the patients' autonomy and beneficence, particularly in the context of serious illness. Some Middle Eastern cultures prioritise beneficence over patient autonomy. This may be used as a justification when patient's family takes over the decision-making process. Although guidelines and protocols regarding information disclosure are fast evolving, there are no sufficient data regarding the application of these guidelines in the clinical context. The objective of this study is to explore the truth disclosure practices of physicians in Bahrain.
\end{abstract}

Method: In this cross sectional study, a random sample of 234 physicians was obtained from the database of Salmaniya Medical Complex (the largest public hospital in Bahrain). We used self-administered 21-item questionnaire to assess the practices and attitudes of physicians regarding disclosure of information to patients with serious illnesses.

Results: A total of 200 physicians completed the questionnaire with a response rate of $69.6 \%$. The question about the usual policy of disclosure revealed that $62.5 \%$ (125) of the doctors would always disclose the diagnosis to the patients, $26 \%$ (52) would often disclose the diagnosis and only $1 \%$ would never disclose the real diagnosis to a competent adult. Only $15 \%$ of the physicians would never make exceptions to their policy of "telling the patient" while all remaining physicians (85\%) made exceptions to their policy either often, occasionally or rarely. The most common reason for not disclosing the diagnosis was family request (39.5\%). About $64.5 \%$ of the physicians were not aware of any existing protocol or policy for diagnosis disclosure to patients. There was no statistically significant association between doctors' policy of disclosure and other demographic variables.

Conclusion: Most physicians opt to disclose the truth; however, the majority would make exceptions at some point particularly upon family request. Regional truth disclosure policies should take into consideration the interplay and balance between patient autonomy and the role played by the family in the decision-making process.

Keywords: disclosure, truth, diagnosis, ethics, breaking news

\section{Introduction}

Confidentiality, the foundation of the doctor-patient relationship, is a key part of respecting the patient's autonomy, one of the four traditional pillars of medical (Beauchamp \& Childress, 2013). Autonomy implies the capacity to think, decide and act on one's own free initiative (British Medical Association., 2013). In the Western culture, this is highly valued by both healthcare providers and patients, who are usually well informed about this topic, which ultimately lead to gradual attenuation of medical paternalism (Gilbar \& Miola, 2015; Gillon, 1994; Khoury \& Khoury, 2015; Surbone, 2006; Tuckett, 2004). However, in some Middle Eastern cultures, beneficence usually precedes autonomy. Breaking the news to the patient might be considered harsh and it is expected that family members take over the decision-making process especially in the context of serious illness (Al-Mohaimeed \& Sharaf, 2013; Bou Khalil, 2013; Hamadeh \& Adib, 1998; Harrison et al., 1997; Mobeireek et al., 2008; Rodriguez Del Pozo et al., 2012).

Multiple studies conducted across the Middle East showed cross-cultural differences in truth-telling attitudes and practices by physicians, further highlighting the variation in emphasis on patient's autonomy and beneficence 
(Al-Mohaimeed \& Sharaf, 2013; Bou Khalil, 2013; Hamadeh \& Adib, 1998; Harrison et al., 1997; Mobeireek et al., 2008; Rodriguez Del Pozo et al., 2012).

In 1998, a study in Lebanon showed that only $47 \%$ of Lebanese physicians disclosed cancer diagnosis directly to the patient (Hamadeh \& Adib, 1998). Nearly a decade later, a Saudi study showed that $56 \%$ of the Saudi physicians would disclose bad news directly to the patients even if that was against the family wishes (Mobeireek et al., 2008). In 2012, a Qatari study showed that $90 \%$ of the physicians would disclose cancer diagnosis to the patients but $66 \%$ of them said they may make exceptions to that rule (Rodriguez Del Pozo et al., 2012).

On the opposite side, public's point of view was also investigated. In Lebanon, $42 \%$ of respondents were against direct disclosure to the patient (Hamadeh \& Adib, 1998). In the above-mentioned Saudi study, $49 \%$ of respondents would prefer bad news be told to the patient directly despite the family's objections to direct disclosure (Mobeireek et al., 2008).

There are scarce published studies from the Kingdom of Bahrain about truth telling among physicians hence, we conducted this cross-sectional study in a diverse cohort of physicians to assess physicians' attitudes and practices towards diagnosis disclosure in the context of serious illness.

\section{Method}

\subsection{Design}

A descriptive cross-sectional study was conducted to assess physicians' attitudes and practices towards truth telling while encountering patients with serious illnesses. Ethics approval was obtained from the Royal College of Surgeons in Ireland - Medical University of Bahrain Research Ethics Committee and Ministry of Health.

\subsection{Participant (Subject) Characteristics}

A random sample of 234 physicians was obtained from the total list of physicians. The exclusion criteria include specialties that either have no direct interaction with the patient in terms of explaining and discussing the final diagnosis, e.g. "pathologist and radiologist" or who commonly deal with patients who are incapable of giving informed consent, such as children and those with mental disorders (paediatricians and psychiatrics).

\subsection{Sampling Procedures and Setting}

We obtained a list of all residents, chief residents, specialists and consultants working at Salmaniya Medical Complex. We excluded non eligible physicians from the list and then, we took a randpme computer-generated sample of 234 physicians.

The study was conducted at Salmaniya Medical Complex (SMC) the largest public hospital in Bahrain with a capacity of nearly 1,200 beds.

\subsubsection{Sample Size, Power, and Precision}

A sample of 234 is sufficient to provide us information within 5\% margin of error; using the following equation:

$\mathrm{X}=\mathrm{Z}_{\alpha / 2}{ }^{2} * \mathrm{p} *(1-\mathrm{p}) / \mathrm{MOE}^{2}$, and $\mathrm{Z}_{\alpha / 2}$ is the critical value of the Normal distribution at $\alpha / 2$ (e.g. for a confidence level of $95 \%, \alpha$ is 0.05 and the critical value is 1.96 ), MOE is the margin of error, $p$ is the sample proportion, and $\mathrm{N}$ is the population size.

\subsubsection{Measures and Covariates}

The questionnaire was adapted from a previous study and modified for use in the present study (Naji, Hamadeh, Hlais, \& Adib, 2015). The same tool has been used on 86 physicians in a parallel study in Jordan (Borgan, Amarin, Othman, Suradi, \& Qwaider, 2018). The questionnaire was reviewed by two external experts and piloted on a sample of twenty physicians. The reliability of the questionnaire was tested and showed a chronbach's alpha value of 0.87 .

Our definition of serious illness included a terminal illness (for example, metastatic cancer), a slowly progressive, life-threatening illness (for example, AIDS), or an adequately treatable condition with a high risk of mortality (for example, myocardial infarction).

\section{Results}

\subsection{Statistics and Data Analysis}

Data were entered into the Statistical Package for Social Sciences (SPSS). Descriptive statistics were generated fro all items. Pearson's Chi square test was used to test the association between disclosure policy and other demographic variables. A p-value of $<0.05$ was considered statistically significant. 


\subsection{Baseline Data}

A total of 200 doctors completed the questionnaire giving an overall response rate of $69.6 \%$. Males constituted $56 \%$ (112) of the sample population. The mean (SD) age was 38.2 (9.22) years. Majority of participants were engaged or married $(79.5 \%$; 159$)$. Although Bahrainis made up to $83.5 \%$ (167) of the participants, $72 \%$ (144) of the total sample had graduated from outside Bahrain. About $28 \%$ graduated from the two medical schools located in Bahrain; 21\% (42) from Arabian Gulf University (AGU) and 7\% (14) from the Royal College of Surgeons in Ireland-Medical University of Bahrain. Residents and chief residents formed $74.5 \%$ and $28.5 \%$ of the total sample respectively, specialists were $13 \%$ of the total sample and consultants formed $11 \%$. The mean (SD) years of practice was 11.75(9.22) years (Table 1). The participants' specialities are presented in figure 1 and shows that a significant percentage of respondents came from general surgery and internal medicine departments $(12.5 \% ; 25)$ and $(10 \% ; 20)$ respectively.

Table 1. Characteristics of particpants $(\mathrm{n}=200)$

\begin{tabular}{ll}
\hline Variable & $\mathbf{\%}(\mathbf{N})$ \\
\hline Gender & $56 \%(112)$ \\
Male & $44 \%(88)$ \\
$\quad$ Female & \\
\hline Nationality & $83.5 \%(167)$ \\
$\quad$ Bahraini & $16 \%(32)$ \\
$\quad$ Non-Bahraini & \\
\hline Marital Status & $17.5 \%(35)$ \\
$\quad$ Single & $79.5 \%(159)$ \\
$\quad$ Married/Engaged & $2 \%(4)$ \\
$\quad$ Divorced/Widowed & \\
\hline Undergraduate Studies & $21 \%(42)$ \\
AGU & $7 \%(14)$ \\
RCSI-MUB & $72 \%(144)$ \\
Other & \\
\hline Medical Employment Level & $74.5 \%(95)$ \\
Resident & $28.5 \%(57)$ \\
Chief Resident & $13 \%(26)$ \\
Specialist & $11 \%(22)$ \\
Consultant & $42 \%(84)$ \\
\hline Years of Practice & $58 \%(116)$ \\
$\quad<12$ &
\end{tabular}




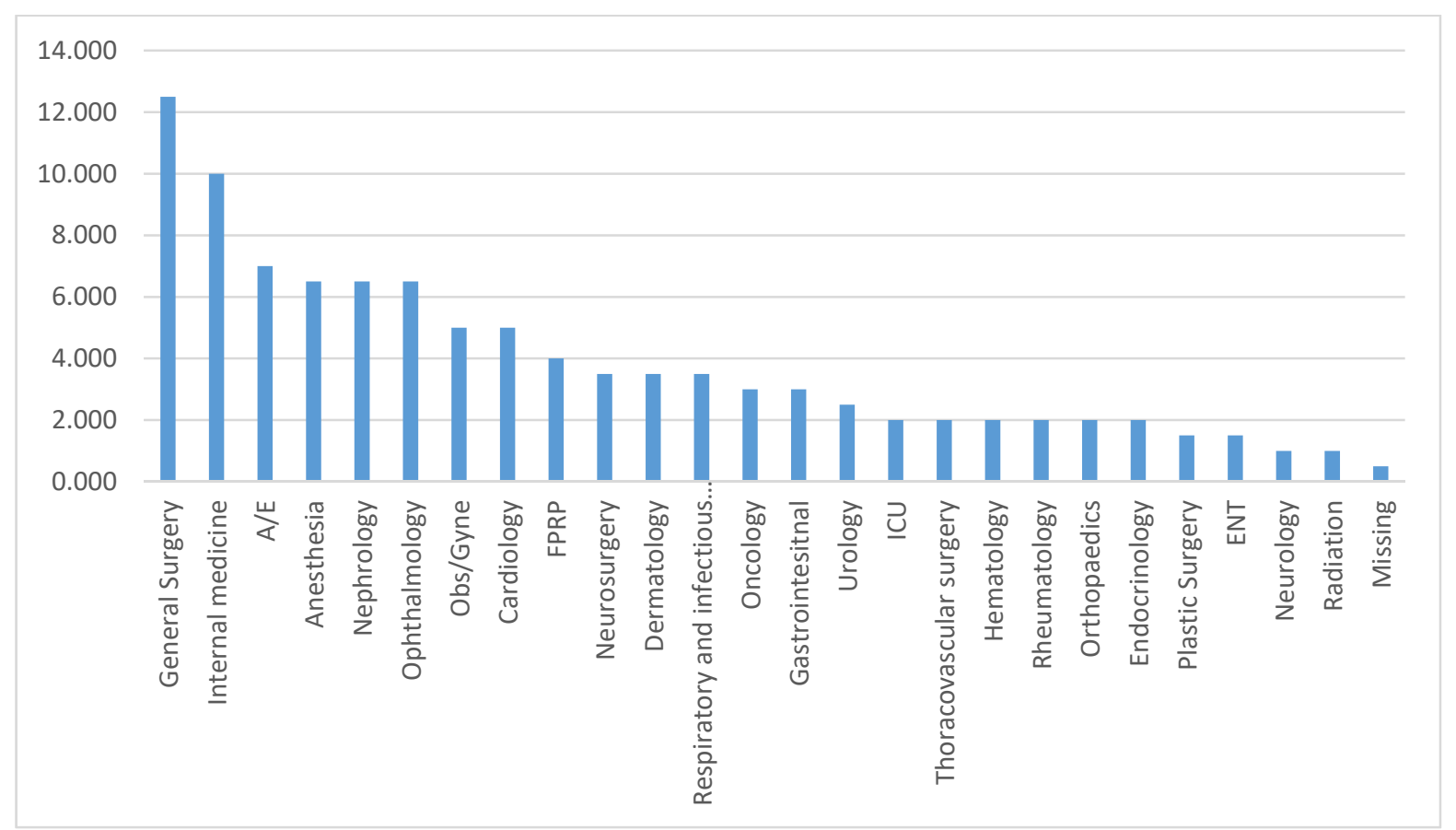

Figure 1. Specialities of Participants $(\mathrm{n}=200)$

About $68 \%$ of the physicians would encounter patients with serious illnesses at least daily or weekly. The question about the usual policy of disclosure revealed that $62.5 \%$ (125) of the doctors would always disclose the diagnosis to the patients, $26 \%$ (52) would often disclose the diagnosis and only $1 \%$ would never disclose the real diagnosis to a competent adult (Figure $2 \mathrm{a}$ ). Only $15 \%$ of the physicians would never make exceptions to their policy while all remaining physicians (85\%) made exceptions to their policy of "telling the patient" either often, occasionally or rarely (Figure 2b).

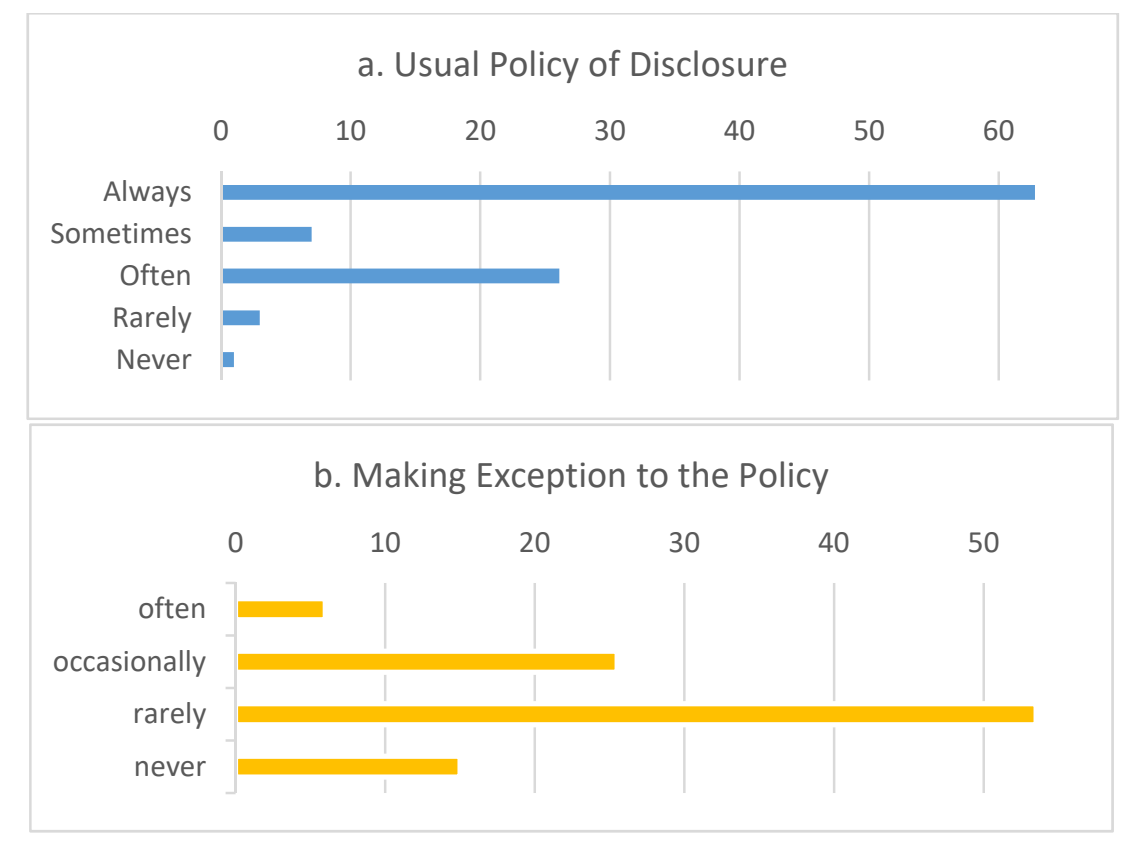




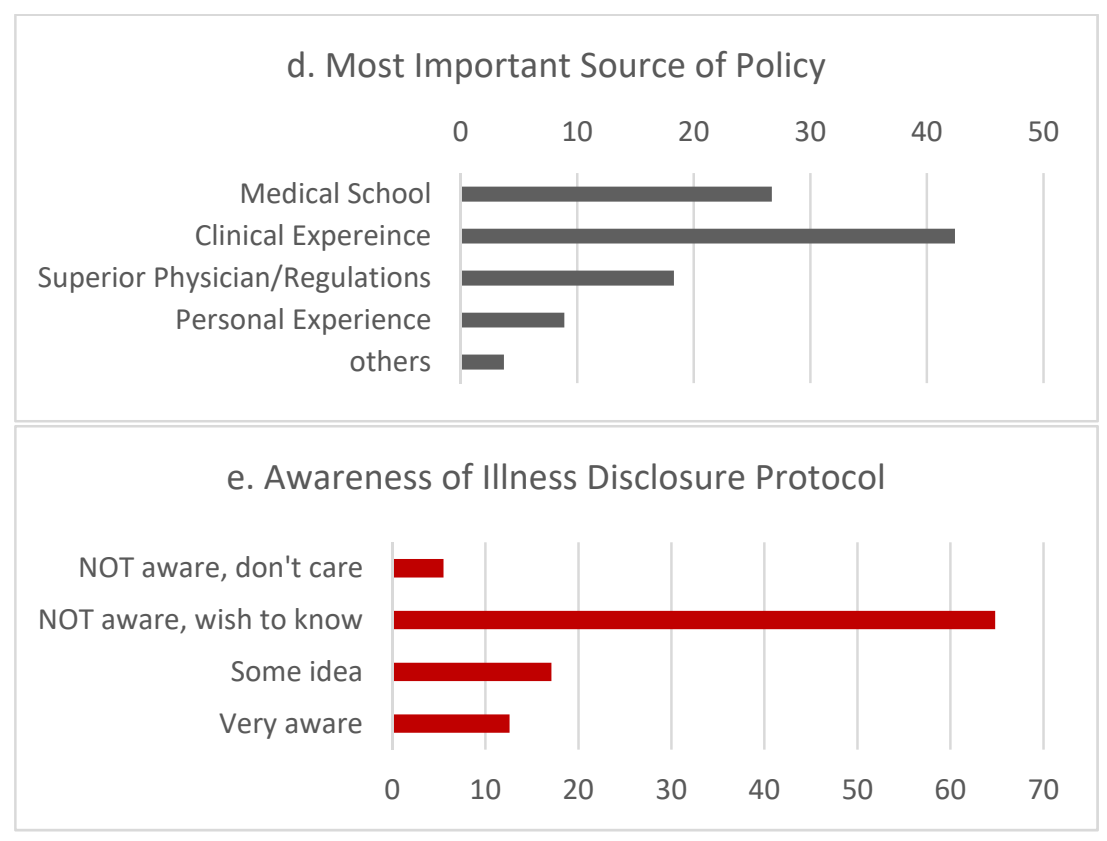

Figure 2. a. Usual policy of disclosure; b. Making exception to the policy; c. Reasons for not disclosing to patients; d. Most important source of policy; e. Awareness of illness disclosure protocol

On further questioning, the most common reason for not disclosing was family request $(39.5 \%), 14 \%$ of the physicians would take this decision by themselves and $15 \%$ would request the patients' consent (Figure $2 \mathrm{c}$ ). The two most important sources of the adopted policy was physicians' clinical experience (40.5\%) and medical school curriculum (25.5\%) (Figure 2d).

About $64.5 \%$ of the physicians were not aware of any existing protocol or policy for diagnosis disclosure to patients in Bahrain. (Figure 2e) and the majority (96\%) believed that hospitals should develop clear policies on how to deal with disclosure issues.

The physicians were given two statements to judge. The first was: "all patients have the right to know their diagnosis" and almost all doctors (97.5\%) agreed with it. The second statement was: "most patients prefer to know their diagnosis" and here too, the majority (84.5\%) agreed with it.

The majority of physicians (96.5\%) thought that, God forbid, if they were patients they would like their doctor to tell them their diagnosis however, $31.8 \%$ thought withholding information might be beneficial to the patients' general health.

The participating physicians were asked to evaluate the importance of 11 factors in determining whether a diagnosis should be told or withheld from the patient. The most important factors were the patient's wish, age, emotional status and patient's educational level. Gender, nationality and religious beliefs were listed as less important.

There was no statistically significant association between doctors' policy of disclosure and other demographic variables such as age (P-value 0.582), gender (P-value 0.572), nationality (P-value0.103), marital status (P-value 0.963 ) and employment post (P-vales 0.877).

\section{Discussion}

This is one of the few reports in the Middle East evaluating the attitudes and practices of physicians regarding the disclosure of information to patients with a serious illness. The main aim is to set the foundation for establishing culturally sensitive guidelines and legislations concerning truth disclosure in the Kingdom of Bahrain. The lack of such legislations in most parts of the Middle East was reported as the main challenge in this area (Bou Khalil, 2013).

Most doctors agreed that there is no clear policy regarding the disclosure of information and also believed that it is important to develop clear policies and training on how to deal with disclosure issues.

Only $62.5 \%$ of the doctors claimed to always disclose full information regarding the disease to the patients. This 
finding remains unchanged when compared to other studies conducted in the region recently as well as studies conducted two decades ago (Al-Mohaimeed \& Sharaf, 2013; Bou Khalil, 2013; Gillon, 1994; Harrison et al., 1997; Mobeireek et al., 2008; Qasem, Ashour, Al-Abdulrazzaq, \& Ismail, 2002; Rodriguez Del Pozo et al., 2012; Tuckett, 2004) . However, compared to the western studies, the level of truth-telling in the USA has jumped from 10 to $97 \%$ of physicians favouring full disclosure in the last 20 year (Kazdaglis et al., 2010).

This physician hesitance in telling the truth is challenged by the discrepancy between family and patients' attitudes regarding truth disclosure. Whilst family members and caregivers may be in favour of "protective" concealment, patients show a steady desire for knowing the truth about their illness (Al-Amri, 2009, 2016).

The patient's wish for knowing the truth was considered the most important factor related to information disclosure as seen by the physicians. Additionally, most physicians agreed that all patients would like to know and have the right to know their diagnosis - showing that the attitude of information disclosure is generally patient driven. However, the main reason for not disclosing information to a patient was "family's request" which is in accordance with the study in Jordan (Borgan et al., 2018). Bahraini culture, just like other Middle Eastern cultures appreciates the role of the family in the illness experience. Although this has a multitude of benefits - providing social and emotional support for example - making decisions concerning a particular patient solely based on the wishes of their family, and without including them, even when patients are fully capable of receiving and processing the information, is considered unethical (British Medical Association, 2013).

The policy of disclosure was consistent all across the board and didn't show significant associations with any sociodemographic variables. This is in agreement with other studies in the region (Borgan et al., 2018).

In this study, the most common reason for nondisclosure is a direct request from the patient's family, without the patient's consent. This finding provides evidence of the role played by family in health decision making. This is identical to the findings from the Jordanian study where a similar culture exists (Borgan et al., 2018).

When deciding to withhold health information from the patient, about 14 per cent of respondents usually made the decision independently. This is congruent with the study from Jourdan and reflects medical paternalism (Borgan et al., 2018). However, the study did not include a question about the impact of patient's gender differences on the disclosure policy.

Strengths of this study include random sampling technique, high response rate and the use of a previously validated structured questionnaire. However, there are a few limitations which need to be addressed. This study did not explore patient's preference about truth disclosure which could be influential in shaping future guidelines. One should note however that this has been studied over the last two decades in other Middle Eastern countries (Hamadeh \& Adib, 1998), where results indicated a striking shift in patient's preference towards knowing the truth.

These findings illustrate the way in which actual medical practice often derives from local medical culture rather than what is taught in textbooks or at medical school. In this case the current Middle Eastern culture regarding disclosure of bad news is reminiscent of the transition of attitudes and behaviours that took place in the west a generation ago. A major force that could inhibit such a transition of practice towards greater disclosure is the closeness and deep continuity of family relationships that exist in Bahrain when compared to a typical western country. This raises the issue of a model of "relational autonomy" as opposed to a traditional western individualistic view of autonomy (Dove, 2017). Whilst relational autonomy cannot generally be used to justify deceit or concealment the underlying cultural assumption that - within a family your business is in fact my business - can easily lead to this if the family feel stressed or distressed by bad news. Clearly, doctors strongly want to know the truth themselves and also usually believe patients have a right to the truth. The disparity between this view and the reality of clinical practice can therefore be understood as culture trumping theory.

The relationship between culture and ethics is complex - they are inextricably bound to each other (Pellegrino, Mazzarella, \& Corsi, 1992). Nevertheless, it would be wrong to see this as an argument for moral relativism. This paper supports the argument that central ethical principles are held in common across different cultures, however culture can influence the degree of importance, or ranking, that we attribute to each principle with respect to the others (Misselbrook, 2017).

\section{Conclusion}

Information disclosure is a crucial and complex topic and the cornerstone to retain the doctor patient's relationship. Protocols, regulations and training are fundamental to protect both patients' and physicians' rights, taking into consideration the interplay and balance between patient autonomy and the decision-making role of family and caregivers when shaping the regional truth disclosure polices. Patients need to be taught to expect truthfulness 
from their doctors, but families also need support to understand why this is almost always appropriate, and how they themselves can deal with the strains and possible distress that truthful disclosure may cause.

\section{Acknowledgments}

We would like to thank all participants for their time and effort.

\section{Competing Interests Statement}

The authors declare that there are no competing or potential conflicts of interest.

\section{References}

Al-Amri, A. M. (2009). Cancer patients' desire for information: a study in a teaching hospital in Saudi Arabia. East Mediterr Health J, 15(1), 19-24. https://doi.org/10.26719/2009.15.1.19

Al-Amri, A. M. (2016). Disclosure of cancer information among Saudi cancer patients. Indian J Cancer, 53(4), 615-618. https://doi.org/10.4103/0019-509X.204771

Al-Mohaimeed, A. A., \& Sharaf, F. K. (2013). Breaking Bad News Issues: A Survey Among Physicians. Oman Medical Journal, 28(1), 20-25. https://doi.org/10.5001/omj.2013.05

Beauchamp T., \& Childress. (2013). J. Principles of Biomedical Ethics (7th ed.). Oxford: Oxford University Press.

Borgan, S. M., Amarin, J. Z., Othman, A. K., Suradi, H. H., \& Qwaider, Y. Z. (2018). Truth Disclosure Practices of Physicians in Jordan. J Bioeth Inq, 15(1), 81-87. https://doi.org/10.1007/s11673-018-9837-x

Bou Khalil, R. (2013). Attitudes, beliefs and perceptions regarding truth disclosure of cancer-related information in the Middle East: a review. Palliat Support Care, 11(1), 69-78. https://doi.org/10.1017/S1478951512000107

British Medical Association. (2013). Medical students ethics toolkit. Retrieved from https://www.bma.org.uk/advice/employment/ethics/medical-students-ethics-toolkit

Dove, E. S., Susan, E. K. Lucivero, F., Machirori, M., Dheensa, S., \& Prainsack, B. (2017). Beyond individualism: Is there a place for relational autonomy in clinical practice and research? Clinical Ethics, 12(3), 150-165. https://doi.org/10.1177/1477750917704156

Gilbar, R., \& Miola, J. (2015). One Size Fits All? On Patient Autonomy, Medical Decision-Making, And The Impact Of Culture. Med Law Rev, 23(3), 375-399. https://doi.org/10.1093/medlaw/fwu032

Gillon, R. (1994). Medical ethics: four principles plus attention to scope. Bmj, 309(6948), 184-188. https://doi.org/10.1136/bmj.309.6948.184

Hamadeh, G. N., \& Adib, S. M. (1998). Cancer truth disclosure by Lebanese doctors. Soc Sci Med, 47(9), 1289-1294. https://doi.org/10.1016/S0277-9536(98)00203-2

Harrison, A., al-Saadi, A. M., al-Kaabi, A. S., al-Kaabi, M. R., al-Bedwawi, S. S., al-Kaabi, S. O., \& al-Neaimi, S. B. (1997). Should doctors inform terminally ill patients? The opinions of nationals and doctors in the United Arab Emirates. J Med Ethics, 23(2), 101-107. https://doi.org/10.1136/jme.23.2.101

Kazdaglis, G. A., Arnaoutoglou, C., Karypidis, D., Memekidou, G., Spanos, G., \& Papadopoulos, O. (2010). Disclosing the truth to terminal cancer patients: a discussion of ethical and cultural issues. East Mediterr Health J, 16(4), 442-447. https://doi.org/10.26719/2010.16.4.442

Khoury, B. S., \& Khoury, J. N. (2015). Consent: a practical guide. Aust Dent J, 60(2), 138-142. https://doi.org/10.1111/adj.12301

Misselbrook, D. (2017). Culture and ethics in primary care. In A. Papanikitas, \& J. Spicer (Eds.), A Handbook of Primary Care Ethics (Chapter 6). London: Taylor and Francis (CRC) Press.

Mobeireek, A. F., Al-Kassimi, F., Al-Zahrani, K., Al-Shimemeri, A., al-Damegh, S., Al-Amoudi, O., . . . Gamal-Eldin, M. (2008). Information disclosure and decision-making: the Middle East versus the Far East and the West. J Med Ethics, 34(4), 225-229. https://doi.org/10.1136/jme.2006.019638

Naji, F., Hamadeh, G., Hlais, S., \& Adib, S. (2015). Truth Disclosure to Cancer Patients: Shifting Attitudes and Practices of Lebanese Physicians. AJOB Empirical Bioethics, 6(3), 41-49. https://doi.org/10.1080/23294515.2014.996265

Pellegrino E., Mazzarella P., \& Corsi P. (1992). Transcultural Dimensions in Medical Ethics. In. US: University Publishing Group Inc, University of Michigan. 
Qasem, A. A., Ashour, T. H., Al-Abdulrazzaq, H. K., \& Ismail, Z. A. (2002). Disclosure of cancer diagnosis and prognosis by physicians in Kuwait. Int J Clin Pract, 56(3), 215-218.

Rodriguez Del Pozo, P., Fins, J. J., Helmy, I., El Chaki, R., El Shazly, T., Wafaradi, D., \& Mahfoud, Z. (2012). Truth-telling and cancer diagnoses: physician attitudes and practices in Qatar. Oncologist, 17(11), 1469-1474. https://doi.org/10.1634/theoncologist.2012-0128

Surbone, A. (2006). Telling the truth to patients with cancer: what is the truth? Lancet Oncol, 7(11), 944-950. https://doi.org/10.1016/S1470-2045(06)70941-X

Tuckett, A. G. (2004). Truth-telling in clinical practice and the arguments for and against: a review of the literature. Nurs Ethics, 11(5), 500-513. https://doi.org/10.1191/0969733004ne728oa

\section{Copyrights}

Copyright for this article is retained by the author(s), with first publication rights granted to the journal.

This is an open-access article distributed under the terms and conditions of the Creative Commons Attribution license (http://creativecommons.org/licenses/by/4.0/). 